\title{
Multidimensional Data Format Specification: A Generalization of the American College of Radiology-National Electric Manufacturers Association Standards
}

\author{
Jayaram K. Udupa, Hsiu-Mei Hung, Dewey Odhner, and Roberto Goncalves
}

\begin{abstract}
Multidimensional image data are becoming increasingly common in biomedical imaging. Three-dimensional visualization and analysis techniques based on three-dimensional image data have become an established discipline in biomedicine. Some imaging problems generate image data of even higher dimensions. It often becomes necessary, rather than just convenient, to consider the higher-dimensional data as a whole to adequately answer the underlying imaging questions. Despite this established need for convenient exchange of image and image-derived information, no exchange protocols are available that adequately meet the needs of multidimensional imaging systems. This paper describes an exchange protocol that has been designed after careful consideration of the common requirements of methodologies for visualization and analysis of multidimensional data. It is based on and is a generalization of the widely accepted American College of Radiology-National Electrical Manufacturers Association (ACR-NEMA) standards specified for two-dimensional images. It is implemented and actively being used in a data-, application-, and machine-independent software environment, being developed in the authors' department, for the visualization and analysis of multidimensional images.
\end{abstract}

Copyright 11992 by W.B. Saunders Company

$\mathbf{M}$ ULTIDIMENSIONAL DATA arise in a variety of disciplines in the biomedical sciences. The data are either produced by an imaging device, such as an imaging scanner, or generated by a simulation procedure. Although three-dimensional (3-D) image data [generated by medical computed tomography (CT), magnetic resonance (MR), positron-emission tomography (PET), and ultrasonography (US) scanners] are the most frequently encountered, higher dimensional data are also becoming available. Some examples of $n$-dimensional $(n \mathrm{D})$ data with $n>3$ that are now available or that can be potentially generated are as follows. CT

From the Department of Radiology, University of Pennsylvania, Philadelphia, $P A$.

Supported by National Institutes of Health Grant CA50851. Address reprint requests to Jayaram K. Udupa, Department of Radiology, University of Pennsylvania, Philadelphia, $P A$.

Copyright $\odot 1992$ by W.B. Saunders Company

0897-1889/92/0501-0009\$03.00/0 or MR volume images of a moving joint or of a beating heart ${ }^{1,2}: n=4$, the independent variables being three spatial and one temporal. MR spectroscopic images ${ }^{3}$ of a beating heart: $n=5$, four independent variables are as in the previous example and the fifth is an MR spectral variable. Dynamic phase-space representation of electroencephalogram signals: $n=4$ to $8,{ }^{4}$ an example of data generated by simulation procedures.

The usefulness of treating 3-D image data holistically rather than as a set of 2-D images has been well established by the developments in 3-D imaging during the past 15 years. ${ }^{5}$ The need for treating higher dimensional data as a whole (rather than as a set of 3-D data) requires some justification because they are not yet as common and established as 3-D data. Of the many examples that illustrate the convenience (such as saving user time, computational time, etc) resulting from such treatment, we give two examples that illustrate the necessity of such treatment. (1) When an $n \mathrm{D}$ data set contains multiple structures, it is crucial to consider each structure in its entirety. Consider a 4-D data set pertaining to a beating heart. The data may contain multiple 3-D dynamic structures such as blood pools in chambers and vessels, myocardium, and so on. The connectedness of each dynamic structure is hard to infer from the substructures presented at each instant. This inference may be an impossible mental exercise when the structure does not conform to known normal configurations, as in congenital heart defects. Considering the structure as a 4-D (connected) entity may be the only approach for the success of visualization and analysis of such structures. (2) There are MR imaging protocols that allow tagging the myocardium magnetically during the imaging process. ${ }^{6}$ This essentially allows affixing a grid system to the myocardium at some initial instant, which then moves with the myocardium during the cardiac cycle. By studying how the grid system distorts, it is possible to perform a comprehensive motion 
analysis of the myocardium. ${ }^{7}$ Unless some simplifying assumptions about the nature of the motion itself are made, this cannot be treated as a 2-D or 3-D image analysis problem.

It is clear that there is a need for a multidimensional data exchange format that fulfills the needs of multidimensional data visualization and analysis. For reasons given earlier, it is just as important to treat multidimensional structures derived from image data holistically as it is to consider image data in that fashion. As multidimensional data and their analysis become more common, there will be a need for exchange of structure data in addition to the need for exchange of image data. No comprehensive protocols exist yet that cater to the needs of multidimensional image visualization and analysis. This article is an attempt to fill this gap.

Although several file exchange protocols exist in the general area of imaging science, ${ }^{8-10}$ and efforts are underway to address the data exchange issue in the more general area of scientific visualization, ${ }^{11}$ we are not aware of any established exchange protocol that has taken into careful consideration the requirements of biomedical visualization and analysis. We have been developing a data-, application-, and machine-independent software environment, called 3DVIEWNIX, ${ }^{12}$ for the visualization and analysis of multidimensional biomedical data. This environment incorporates a variety of methodologies and algorithms and is developed for UNIX machines $^{13}$ supporting the X-Window system. ${ }^{14}$ Because American College of Radiology-National Electrical Manufacturers Association (ACR-NEMA) standards ${ }^{15}$ suggested for the representation, archival, and communication of 2-D images are the most widely accepted protocols within medical imaging, and because their extension and generalization to include multidimensional image and structure data did not pose any intrinsic difficulties, we decided to build on their principles. This paper describes the principles underlying this generalization and the complete generalized specification. We have tried to make the paper self-contained and independent of the description of the ACRNEMA specifications. Readers interested in more details of the ACR-NEMA specification may consult their publication. ${ }^{15}$

\section{TERMINOLOGY}

Data handled by multidimensional imaging systems are mainly of three kinds. The first can be considered to be the digitized and quantized estimate of a vector-valued function of a vector variable. The second and the third represent information derived from the first.

We call the first a scene, the discretized domain of the function the scene domain, the dimension of the independent vector variable the scene dimension $(s d)$, the device that produces an estimate of the function an imaging device, each digitized instance of the independent vector variable a cell, and the quantized value of the function at the cell the cell density. As an example, a stack of slice image data captured by an imaging device such as a CT scanner is a 3-D scene where the function is scalar valued. At one end, a scene may thus represent a time sequence of MR image data pertaining to a dynamic organ such as the human heart, and at the other end it may represent simply a scalar-valued function of a scalar variable. With each scene, we associate a fixed coordinate system $x_{1}, x_{2}, \ldots, x_{\text {sd }}$ called the scene coordinate system. We assume a fixed coordinate system called the imaging device coordinate system to be associated with each imaging device. We assume these systems to be right-handed in the sense of a generalization of a right-handed 3-D coordinate system (ie, if $u_{1}$, $u_{2}, \ldots, u_{\mathrm{sd}}$ are unit vectors along the coordinate axes, we assume $u_{i} \times u_{i+1}=u_{i+2}$, where $1 \leq i \leq$ $s d$ and $u_{s d+1}=u_{1}$ and $u_{s d+2}=u_{2}$ ). Each cell has a unique location in the scene specified by its real coordinates in the scene coordinate system. We note that the unit of measurement may not be the same along the different coordinate axes.

Data derived from scenes may be in many forms. They may represent another scene; for example, a scene resulting from applying to a given scene a variety of scene transformations such as filtering, segmentation, and interpolation. They may represent nonscene structures; for example, a set of cells constituting an opaque/semitransparent object or the boundary of a set of cells. They may also represent display data; for example, a sequence of red, green, blue color-valued 2-D images represent- 
ing renditions of a sequence of views of the surface of an object.

Associated with every structure data set is a right-handed structure coordinate system $y_{1}, y_{2}$, $\ldots, y_{\text {std }}$. We assume that all structures are representable as a tree through hierarchical subdivision, the subdivisions representing natural groupings in the structure. For example, a 3-D object represented as a set of cells can be divided into subsets, each of which represents cells having the same $y_{3}$ coordinate. Each such subset may be further divided into smaller subsets representing cells with the same $y_{2}$ coordinate. Finally, each subdivision of each smaller subset contains the individual cell (ie, the description associated with each cell). This structure represents a slice-by-slice and a rowby-row representation of an arbitrary set of cells. We call the entities represented by the leaf nodes of the tree the terminal structure elements and the entities represented by the intermediate groupings the non-terminal structure elements. In the example just given, the terminal structure elements are the cells; the slice and row groupings constitute the nonterminal structure elements, and the tree has four levels including the root (Fig 1). Most of the structures encountered in multidimensional imaging systems have characteristics similar to those in this example: the tree has a few (two to four) levels; the leaf nodes all occur at the last level; the number of nonterminal structure elements is a small fraction of the total number of terminal structure elements. However, there are other structures, such as the spatial subdivision trees (eg, octrees, quadtrees) that generally do not have these properties.

A display data set derived from a scene is a set of 2-D images that portray information about specific aspects of the objects captured in the scene. In general, the intensity associated with the pixels of the images in the display is vector valued.

In summary, data processed and generated by multidimensional imaging systems are mainly of three kinds: scene data, structure data, and display data. The first constitute mainly data generated by imaging devices or some processed version thereof, the second object information extracted from scenes, and the third a composition of specific object information ready to be displayed.

In designing a data format, one invariably encounters the issue of trade-off between the generality of format and its efficiency. In the data format proposed here, we have tried to keep the generality with as little sacrifice of efficiency as possible. This we achieve by recognizing each situation where data may inherently be of a distinct nature (from the consideration of processing done on the data) and by assigning to it a distinct data type. To do this we identify further data subtypes (IMAGE0, IMAGE1, CURVE0, SURFACE0, SURFACE1, SHELL, etc) among scene, structure, and display data. A specific meaning is imparted to the information stored in a file when we know the data type it represents.

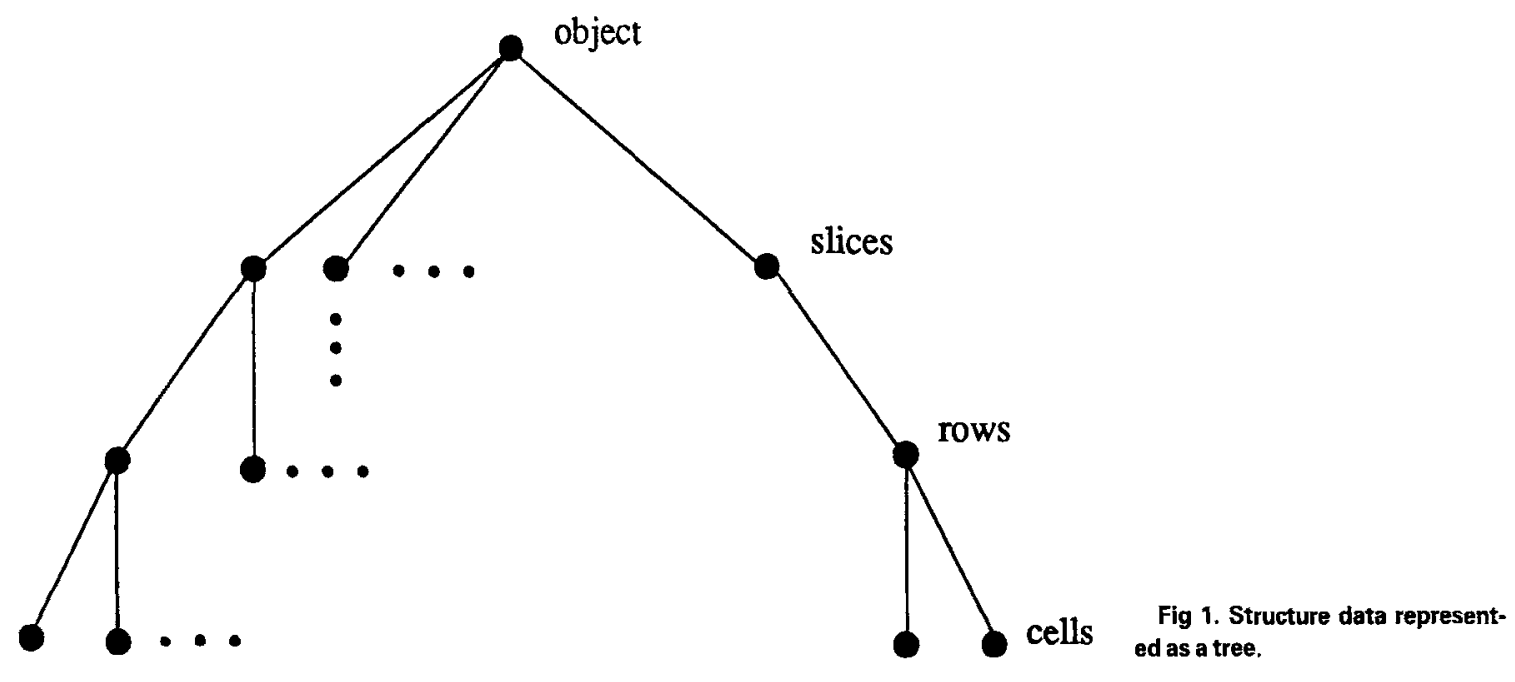


We use the following abbreviations, mostly as used in the ACR-NEMA standards specifications ${ }^{15}$ to describe the data format.

Method of value representation (VR):

BI: 16 bit binary (2's complement), the left byte being more significant than the right byte.

BD: 32 bit binary unsigned, with the bytes appearing in the order byte 1 , byte 0 , byte 3 , byte 2 , where byte $0, \ldots$, byte 3 are in the increasing order of significance. (See LIMITATIONS AND EFFICIENCY.)

GN: Generalized numeric; a set of integer and floating point numbers. The integers are stored first followed by the floating point numbers. The integers may be packed in any specified bit fields and may be signed (2's complement) or unsigned. The floating point numbers may be single or double precision and are stored as per Institute of Electrical and Electronic Engineers standards. ${ }^{16}$

AN: ASCII numeric. An integer number should contain only the characters 0 through 9 , + , and - . A fixed-point number should contain only the characters 0 through 9 , ,+- , and an explicit decimal point (implied decimal points are not permitted). A floating-point number should be as defined in ANSI X3.9 (1978). Character $E$ is permitted to indicate the start of an exponent. When a word consists of two characters, the first should be in the least significant byte. All ASCII fields should be padded if necessary to produce an even number of bytes, the padding character being the space $(20 \mathrm{H})$. The AN text should be right-justified.

AT: ASCII text, storage as for AN except that the AT text should be left-justified.

Definition of value multiplicity (VM): S, single value; $M$, multiple value.

Data element types: 1, type1 (necessary for storage and display of the data); 1D, type1D (type1 with default values); 2, type2 (primary values used for interpretation); 2D, type2D (type 2 with default values); 3 , type 3 (secondary items used for interpretation).

If $\mathrm{VM}$ is $\mathrm{M}$ when $\mathrm{VR}$ is $\mathrm{AT}$ or $\mathrm{AN}$, the delimiter used to separate multiple values is " $\backslash$ ". When VR is BI, BD, or GN, no delimiter is needed and hence not used. (In the latter case, we use delimiters in our descriptions just to show the separation of different value fields.)

\section{DESCRIPTION OF DATA TYPES}

In this section we present complete specifications of scene, structure, and display data.

\section{Scene Data}

Among scene data we identify two typesIMAGE0 and IMAGE1. For IMAGE0 type, we assume that the sampling scheme (ie, the method of digitizing the independent variable) is more systematic, in particular, slice oriented, and matches the way most imaging devices capture scene data. For IMAGE1 type, the sampling scheme is arbitrary.

IMAGE0. We assume the scene domain to be such that (1) the set $S$ of all cells in the scene domain with fixed $x_{i}$ coordinates, for $3 \leq i \leq s d$, to be a 2-D rectangular array, and (2) the distances between consecutive cells in the $x_{1}$ and $x_{2}$ directions are constants (if the units of measurement along $x_{1}$ and $x_{2}$ are different, we assume an appropriate definition of distance between cells in the $\left(x_{1}, x_{2}\right)$ space to be given). This implies that if we consider the cells to constitute points in an sd-dimensional Euclidean space, the array defined by $S$ constitutes a lattice of rectangles (this is equivalent to having rectangular pixels). We call $S$ together with the densities associated with its cells a slice. Often data captured by imaging devices are such that the set $S$ is not a rectangular array. Such data should be appropriately processed to bring them into this slice form. Unlike the domain of the slice, we will soon see that the scene domain itself need not be of a rectangular array form for an IMAGE0 type scene.

For a given $n, 2 \leq n \leq s d$, and fixed numbers $X_{n+1}, X_{n+2}, \ldots, X_{s d}$, an $n$-scene of a scene is the set of cells $\left\{c=\left(x_{1}, x_{2}, \ldots, x_{n}, X_{n+1}, \ldots, X_{s d}\right) \mid c\right.$ is in the scene domain\} together with the associated cell densities. We call $X_{n+1}$ the location of the $n$-scene in the $(n+1)$-scene of the scene, or simply the location of the $n$-scene. The notion of the $n$-scene allows us to associate a natural hierarchical order with any given scene and express its sampling scheme as a tree structure. The root of the tree represents the given scene, a node represents an $n$-scene, and its sons 
represent the $(n-1)$-scenes. The leaf nodes represent the 2-scenes (slices) of the scene. Figure 2 shows an example of a 4-D scene with four 3-scenes. The four 3-scenes are composed of two, two, three, and three 2 -scenes. Clearly the leaf nodes all appear at the same level of the tree and therefore the tree can be described by a list of the number of sons for each node in the breadth-first order. For the example, this list contains $4,2,2,3,3$. We use this form of tree description to store the number of sons for each node, and except for the root, also the location of the $n$-scene associated with each node.

$I M A G E 1$. No fixed sampling discipline is assumed for IMAGE1 type of scenes. The location and density values for cells are stored explicitly in the scene data group. (Therefore, message items describing the sampling scheme are not relevant for IMAGE1 type scene data.)

\section{Structure Data}

A structure is usually derived from a given scene. We assume that associated with every structure is a structure domain, which is usually a subset of the scene domain. For example, if the structure is a 3-D surface, the structure domain is an appropriate 3-D region that encloses the surface; if the structure is a semitransparent volume, the structure domain is the region associated with the volume. We assign a dimensionality (std) to every structure, which is simply the dimensionality of the structure domain. It is possible that the dimensionality of the scene and of the structure derived from it may be different. For example, the structure may be the 3-D surface of a dynamic object at a particular instance, derived from a 4-D (time-varying) scene. It is not necessary that a structure be always derived from a scene; instead it may be synthesized independently of any underlying scene; for example, an isodose surface in radiation therapy planning or the surface of a prosthesis. In such cases we assume that the structure domain is an appropriate subset of an $s d$ dimensional Euclidean space.

Associated with every structure is a location (specified by an $s d$-dimensional vector and an orientation (specified by std unit vectors each of dimensionality $s d$ ), which respectively represent the location of the origin and orientation of the axes of the structure coordinate system with respect to the imaging device coordinate system. This information is helpful in determining the location and orientation of the structure with respect to the imaging device during visualization and analysis of the structure. This in turn makes possible the comparative and composite study of objects longitudinally and/or from multimodality scene information.

The sampling scheme associated with a structure describes how the structure domain is discretized. This information, specified by the number of samples and their location, is needed in both visualization and analysis of the structure. We assume that the structure domain is discretized in a fashion similar to how the scene domain is discretized for IMAGE0 type scenes, and so the number of samples and their location for the structure should be interpreted exactly as the number of $n$-scenes and their location, described under IMAGE0.

The hierarchical description of a structure as a tree together with the associated terminal (TSE) and nonterminal structure elements (NTSE) (see previous section) is not complete unless the descriptors associated with these

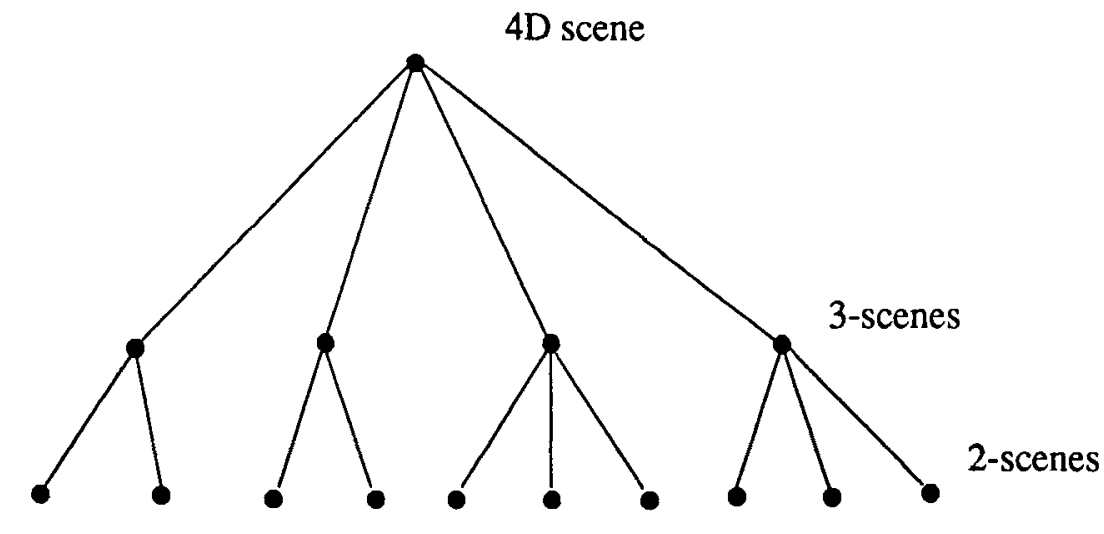

Fig 2. An example illustrating how a 4-D scene is structured using 3- and 2-scenes. 
elements are specified. We associate with each terminal and nonterminal structure element an $n c t$-tuple TSE and an ncnt-tuple NTSE that specify these descriptors. All structure data are stored identically as per the general specification. However, it is the type description that gives a specific interpretation to any given structure data. We describe here some data types; others can be included as required using similar principles.

CURVE0. One method of representing a multidimensional object is by the set of its 2-D boundaries or contours resulting from the intersection of a set of planes with the object. (This structure is commonly used for prosthesis design and manufacture.) Given a scene containing information about the object, the contours are obtained by processing every slice of the scene. ${ }^{5}$ A contour in a slice is a boundary of the object in the slice expressed as a sequence of edges of cells (pixels) that forms a closed curve. ${ }^{5}$ Figure 3 shows an example. CURVE0 is a structure that represents a set of such contours.

The tree representing the CURVE0 structure of an std-dimensional object consists of std +1 levels, as illustrated in Fig 4 for the scene considered in Fig 2. The nonterminal nodes represent the $n$-scenes and the contours, and the terminal nodes represent contour elements. If an $n$-scene does not have any contour specified for it, it is not represented in the tree. The vectors NTSE and TSE associated with the nonterminal and terminal nodes are

$$
N T S E=(n s), T S E=\left(y_{1}, y_{2}, d i r\right),
$$

where $n s=$ the number of sons for the node, $y_{1}$, $y_{2}=$ the coordinates of the cell to which the
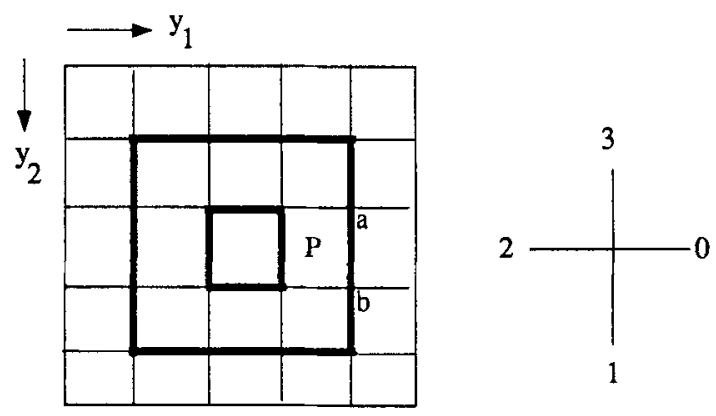

Fig 3. A slice with two contours (bold). A contour element is a line segment such as ab that is specified by the cell $P$ of the object to which it belongs and the direction (0) indicating where the line segment lies with respect to the cell. contour element belongs, and dir = the direction of the contour element relative to the cell.

CURVE1, CURVE2, ..., are type names reserved for other types of boundary curves; for example, for curves that give smoother representations of the contour.

SURFACE0. This structure is used for describing the boundaries of an std-dimensional object as sets of (std-1)-dimensional cells, each boundary forming a closed, connected surface in the std-dimensional space. ${ }^{17} \mathrm{~A}$ file storing the SURFACE0 structure may contain more than one boundary. In the 3-D case, for example, the cell may be considered to be a cuboid and a boundary is a closed and connected surface made up of faces of the cuboids. ${ }^{18}$ A 3-D object may have more than one surface and some or all of these may be stored as a single message. ${ }^{19}$

The hierarchy in the SURFACE0 structure is as shown in Fig 5 . Every boundary element (bel for short) is represented by the cell in the object to which the bel belongs and a direction (as in Fig 3 ) indicating where the bel is with respect to the cell. Because there are $2 \times$ std possible such directions, the bels may be grouped into $2 \times$ std sectors, each sector containing bels of the same orientation. The vectors NTSE and TSE for SURFACE0 are NTSE $=(n s)$, and TSE $=\left(y_{1}\right.$, $\left.y_{2}, \ldots, y_{s t d}, n_{1}, n_{2}, \ldots, n_{s t d}\right)$, where $n s=$ the number of sons for the node, $y_{1}, y_{2}, \ldots, y_{s t}=$ the coordinates of the cell containing the bel, and $n_{1}, n_{2}, \ldots, n_{s t d}=$ the components of a unit surface normal vector associated with the bel.

SURFACE1. This structure is used for describing the 3-D boundaries of a 3-D object as sets of triangular elements (there are several methods that generate such descriptions. ${ }^{20 \cdot 22}$ ) As in SURFACE0 type, a file storing a SURFACE1 type boundary may contain more than one boundary. The hierarchical division of the SURFACE1 structure is as in Fig 5 where each bel is a triangle and each sector represents a set of triangles that represent the part of the boundary surface that lies between two successive slices. The descriptions NTSE and TSE are as follows: $N T S E=(n s), T S E=\left(y_{11}, y_{12}, y_{13}, y_{21}\right.$, $y_{22}, y_{23}, y_{31}, y_{32}, y_{33}, n_{1}, n_{2}, n_{3}$ ), where $n s=$ the number of sons for the node, $y_{11}, y_{12}, y_{13}=$ the coordinates of the first vertex of the bel, $y_{21}, y_{22}$, $y_{23}=$ the coordinates of the second vertex of the 


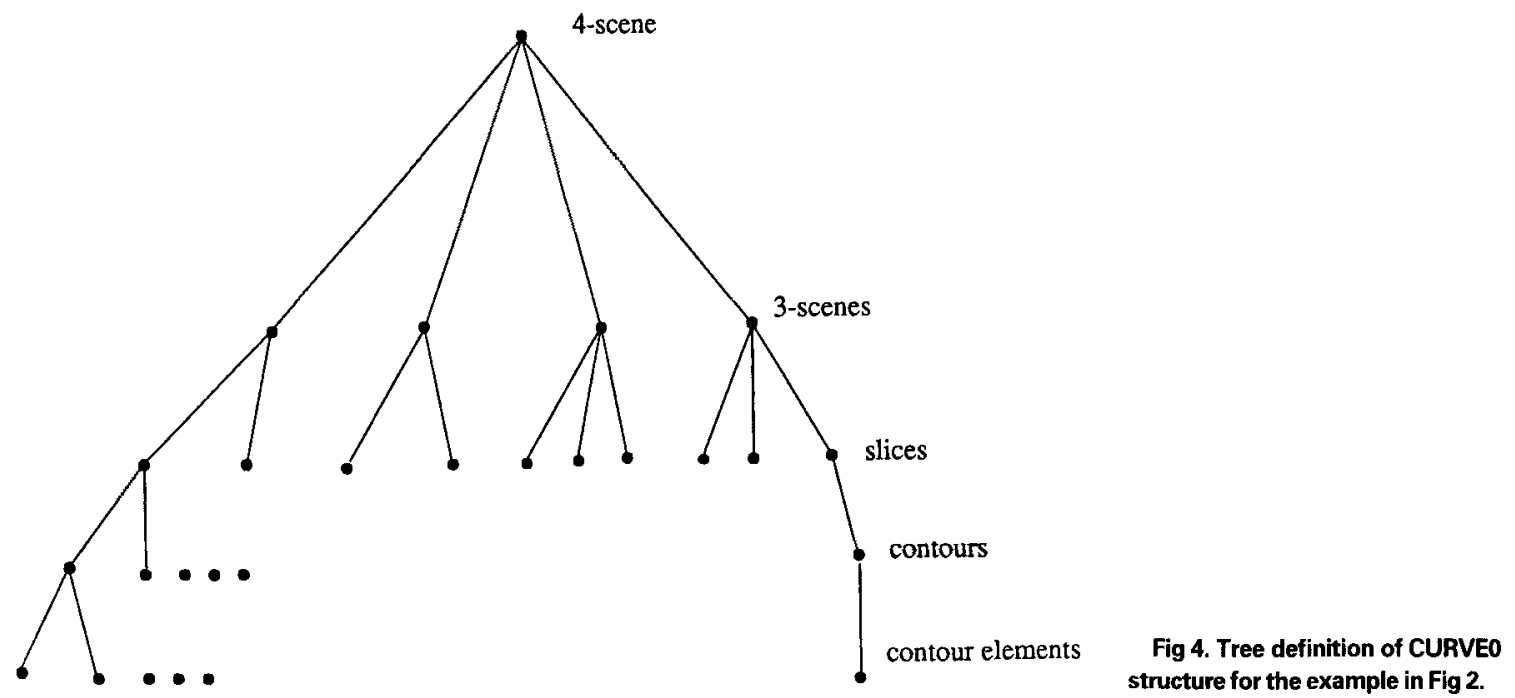

bel, $y_{31}, y_{32}, y_{33}=$ the coordinates of the third vertex of the bel, $n_{1}, n_{2}, n_{3}=$ the components of $a$ unit surface normal vector associated with the bel. We reserve SURFACE2, SURFACE3, ..., type names for other forms of surface description.

SHELL. SHELL is a structure used by processes that allow rendering objects, altering them (for the purpose of simulating surgical manipulations), and measuring them, using both surface $^{23}$ and volume rendering techniques. ${ }^{24-27}$ The structure is used at present only for 3-D objects (although its concepts generalize to higher dimensions), and therefore we describe here only the 3-D version.
The tree specification for SHELL is as shown in Fig 6. An object here is considered to be a set of cells each with a number of descriptors assigned to it. The cells are organized in the slice and row order. The vectors NTSE and TSE associated with the nodes corresponding to nonterminal and terminal structure elements and their descriptions are as follows: NTSE $=$ (ns), TSE = (ncode, $\left.y_{1}, t t, n_{1}, n_{2}, n_{3}, g m, o p, p g\right)$, where

$n s \quad=$ number of sons for the node

ncode = a 6-bit number (value 0 to 63) indicating which of the 6-neighbors (immediate neighboring cells in the $y_{1}, y_{2}$, $y_{3},-y_{1},-y_{2},-y_{3}$ directions) of the

Fig 5. Tree definition for SURFACEO structure.

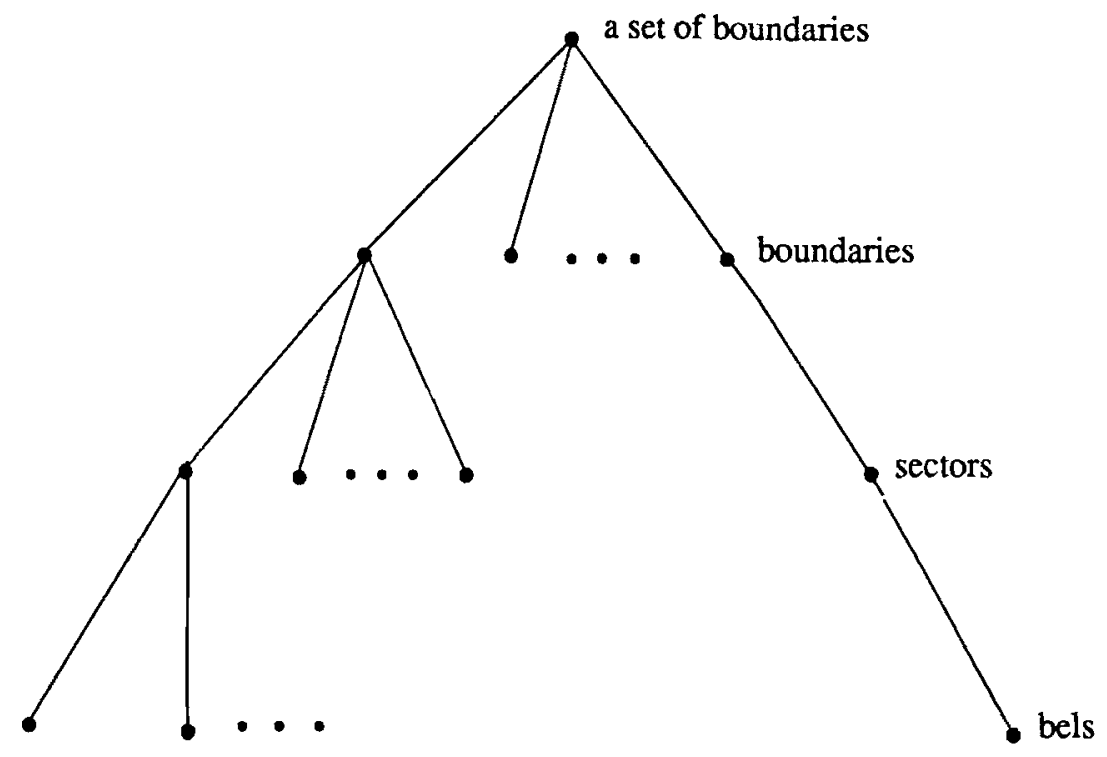




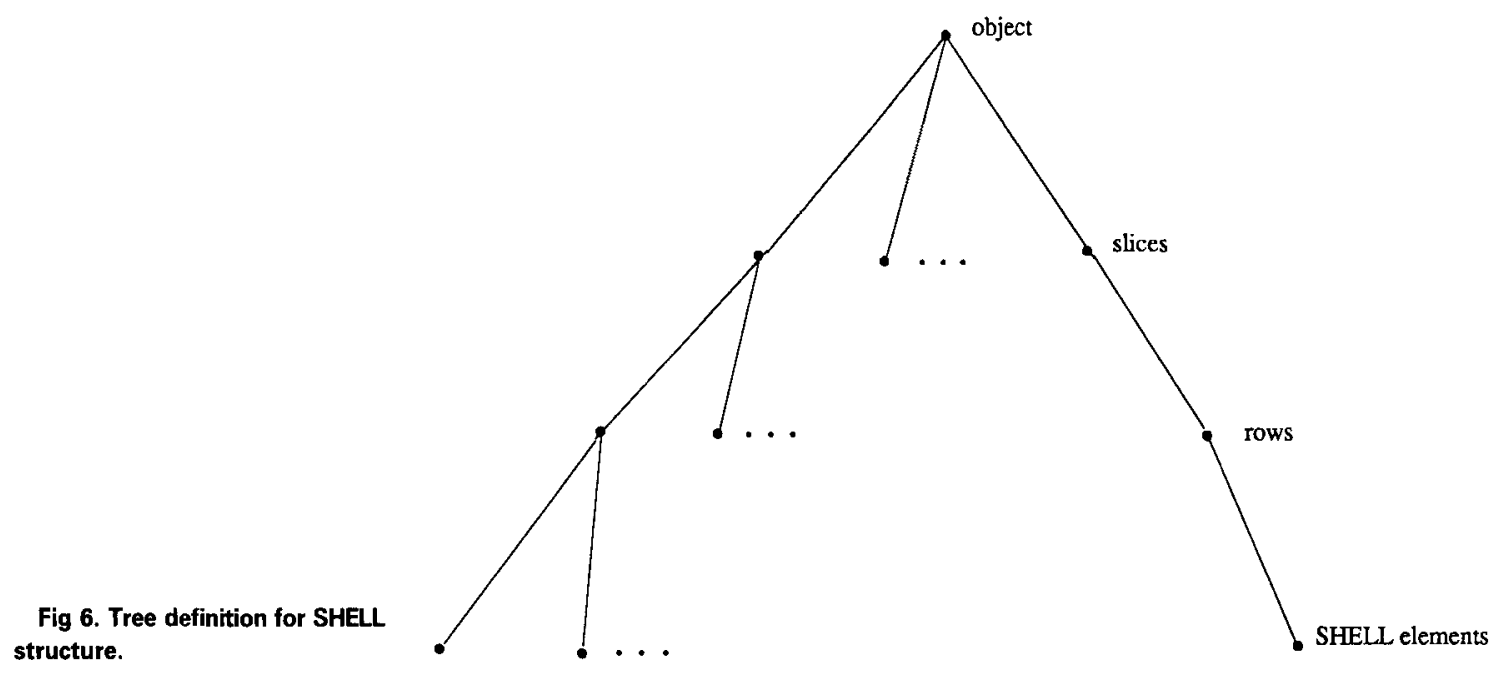

cell have an opacity greater than or equal to a fixed high opacity value $o p_{h}$ (bit set to 1) and which have an opacity less than $o p_{h}$ (bit set to 0 ). If all 6 bits are 1 or if the opacity of the cells is less than a fixed low opacity value $o p_{l}$, the cell is considered not to be a SHELL element and hence not stored. When $o p_{h}=o p_{i}$, we get a binary SHELL

$y_{1} \quad=y_{1}$ coordinate of the cell;

$t t \quad=$ tissue type: 0 , do not care; 1 , air only; 2, air soft tissue mixture; 3 , soft tissue only; 4 , soft tissue and fat mixture; 5 , fat only; 6 , fat and bone mixture; 7 , bone only

$n_{1}, n_{2}, n_{3}=$ a vector normal to the object boundary at the center of the cell; $n$ is 0,1 , $2,3,4$, or 5 depending on which of the six faces of the cell facing respectively the $y_{1}, y_{2}, y_{3},-y_{1},-y_{2},-y_{3}$ directions is intersected by a line drawn from the center of the cell in the direction of the vector; $n_{1}=6$ means that the normal is not well determined; $n_{2}, n_{3}$ are the coordinates of the point of intersection of the line and the face within the face

gm = magnitude of the gradient (scaled relative to the minimum and maximum gradient values) of that scene from which the SHELL is derived, evaluated at the cell using an appropriate digital gradient operator $o p \quad=$ opacity value assigned to the cell;

pg = tissue percentage (in case of mixture, percentage of the higher density tissue in the descriptor of $t t$ ).

It is not necessary to store all of the above information for all rendering techniques. For example, in surface rendering using a binary SHELL, ${ }^{23}$ it is not necessary to store $g m, t t, o p$, and $p g$. The proposed protocol is general enough to handle a variety of such situations.

\section{Display Data}

Each image in a display data set contains a portrayal of some specific aspects of the object information captured in a scene that is ready to be displayed. Sometimes it is possible to parameterize these aspects. We associate a dimensionality $(d d d)$ with each display data set that constitutes the number of independent parameters characterizing object information captured in the images plus 2 (for the dimensionality of each image). The purpose of identifying the number of independent parameters is to allow the viewer to control the way the values of the parameters are changed in order to decide the order in which images in the display data are presented to the viewer. If such control is not desired or needed, the multiplicity of the parameters can be ignored, resulting in only one independent parameter (and in a dimensionality of three). The only order of presenting the images in the latter case is the natural order in which the images occur in the display data stored as a message. Some examples of display 
data that may have a dimensionality greater than three are as follows: 1. Each image is a rendition of an object surface for a given view point. If the viewing direction is described by three angles, and if all angles are to be controlled independently, the images comprising the display data can be characterized by three independent parameters. All connected paths in such a 3-D parameter space correspond to valid and possible sequences of displaying the images. Display data dimensionality is thus five. 2. Each image is a rendition as already described, but of a time-varying surface so that time instance becomes the fourth independent parameter. Possible sequences of images now correspond to connected paths in a 4-D parameter space. Display data dimensionality now is six. 3 . In addition to these four parameters, we may have up to three additional parameters that specify the changing location of a segment of the object that is separated from the rest of the object and that is being interactively moved by a user. 4. Possible additional parameters are those related to object specification; for example, the object specified by a threshold value of scene density. The display data may constitute the different views of a time-varying object as the threshold value is changed $(d d d=7)$.

Associated with every display data set is a right-handed display-data coordinate system $z_{1}$, $z_{2}, \ldots, z_{d d d}$. Associated with every image of the display data is a parameter vector that has $d d d-2$ components, each of which corresponds to one of the independent parameters that characterize the image. Associated with every pixel of the image is an intensity vector that stores the values associated with the pixels.

MOVIEO. This is a particular type of display data that represents cine sequences of surface and volume renditions. The image intensity vector $I V$ is $1-\mathrm{D}$ or $3-\mathrm{D}$, and the parameter vector $P V$ may contain any (finite) number of components. The meaning associated with the components is not fixed and is left to the user to determine (see next section). The examples given earlier indicate some possible meanings. $I V=(i v)$ or $\left(i v_{1}, i v_{2}, i v_{3}\right), P V=\left(p_{1}, p_{2}, \ldots, p_{d d d-2}\right)$, where $i v 1=$ red component, $i v 2=$ green component, $i v 3$ = blue component; when $I V$ is scalarvalued, the value represents pixel gray value.

\section{FORMAT DESCRIPTION}

A message is a set of related data items and each data file contains one message. Each item in a message is assigned a group number and an element number and is completely represented in the file by storing its GROUP\#, ELEMENT\#, LENGTH, and VALUE, where LENGTH is the number of bytes of storage required for the item and VALUE is its actual value. A file thus contains a sequence of such quadruples. If the message is a 2-D scene, for example, some message items (quadruples) store information related to the description of the scene such as its dimension, size, resolution, and so on, and the quadruple associated with group 7FE0 (Table 1) stores the actual pixel density values. The even-numbered groups are called standard groups and are reserved by the ACR-NEMA standards committee. The oddnumbered groups are called shadow groups and may be used for private purposes. All type-1 and type-1D items from standard groups must be present in the message.

The detailed format of storing the items in a message is presented in Table 1. All entries under Group, Element and Length are hexadecimal numbers. Groups numbered 0009, 0029, $002 \mathrm{~B}, 002 \mathrm{D}$, and 8001 do not exist in the 2-D ACR-NEMA standards ${ }^{15}$ and have been created to accommodate multidimensional scene, structure, and display data. The meaning of GROUP:0000, ELEMENT:0800, GROUP: 0008, ELEMENT:0041, and of GROUP:7FE0 have also been broadened. In addition, we have created a new value representation (VR) type called generalized numeric (GN) that allows storing sets of mixed integer and floating point numbers for message items, where the integers may be bit-packed in any specified fashion and the floating point numbers may be single or double precision.

Messages representing data of all types contain message items up to and including GROUP: 0028. Beyond GROUP:0028, message items are included based on their relevance to the data type. Message items relevant to each of the scene, structure, and display data types are as follows: scene $=$ groups up to GROUP:0028 and GROUP:0029, GROUP:7FE0; structure = groups up to GROUP:0028 and GROUP:002B, 
Table 1. Detailed Data Format Specification

\begin{tabular}{|c|c|c|c|c|c|c|c|c|}
\hline Group & Element & \multicolumn{2}{|c|}{ Length } & Value & VR & VM & Type & Description \\
\hline \multicolumn{9}{|c|}{ Command information group } \\
\hline 0000 & 0000 & 0004 & 0000 & - & $\mathrm{BD}$ & $\mathrm{s}$ & 1 & $\begin{array}{l}\text { Group length (an even number of bytes from } \\
\text { the end of the value field to the beginning } \\
\text { of the next group) }\end{array}$ \\
\hline 0000 & 0001 & 0004 & 0000 & - & $\mathrm{BD}$ & $\mathrm{S}$ & 1 & $\begin{array}{l}\text { Message length (an even number of bytes } \\
\text { from the end of the value field to the be- } \\
\text { ginning of the next message) }\end{array}$ \\
\hline 0000 & 0010 & - & - & 一 & AT & S & 1 & $\begin{array}{l}\text { Recognition code (used for identifying the } \\
\text { version number of this protocol) }\end{array}$ \\
\hline 0000 & 0100 & 0002 & 0000 & - & $\mathrm{BI}$ & $s$ & 1 & Command field \\
\hline 0000 & 0110 & 0002 & 0000 & - & $\mathrm{BI}$ & $\mathrm{s}$ & 1 & Message ID \\
\hline 0000 & 0120 & 0002 & 0000 & - & $\mathrm{BI}$ & s & 1 & Message ID being responded to \\
\hline 0000 & 0200 & - & - & - & AT & s & 1 & Initiator \\
\hline 0000 & 0300 & - & -- & - & AT & s & $1 \mathrm{D}$ & Receiver \\
\hline 0000 & 0400 & - & - & - & AT & $s$ & 1 & FIND location \\
\hline 0000 & 0600 & - & - & - & AT & $s$ & 1 & MOVE \\
\hline 0000 & 0700 & 0002 & 0000 & - & $\mathrm{Bl}$ & $s$ & $1 \mathrm{D}$ & Priority \\
\hline \multirow[t]{2}{*}{0000} & 0800 & 0002 & 0000 & - & $\mathrm{BI}$ & $s$ & 1 & Data set type: \\
\hline & & & & & & & & $\begin{array}{l}\text { Scene data: } \\
\text { IMAGE0 }=0 \\
\text { IMAGE1 }=1 \\
\text { Structure data: } \\
\text { CURVE0 }=100 \\
\text { SURFACE0 }=110 \\
\text { SURFACE } 1=111 \\
\text { SHELL }=120 \\
\text { Display data: } \\
\text { MOVIEO }=200 \\
\text { Other data: } \\
\text { GRAPHICS }=2 \text { (see [15]) } \\
\text { TEXT = } 3 \text { (see [15]) }\end{array}$ \\
\hline 0000 & 0850 & 0002 & 0000 & - & $\mathrm{Bl}$ & $s$ & 1D & Number of matches \\
\hline 0000 & 0860 & 0002 & 0000 & - & BI & $s$ & $1 \mathrm{D}$ & Response sequence number \\
\hline 0000 & 0900 & 0002 & 0000 & - & $\mathrm{BI}$ & $\mathrm{S}$ & 1 & Status \\
\hline 0000 & 4000 & - & - & - & AT & $S$ & $1 \mathrm{D}$ & DIALOG receiver \\
\hline 0000 & 4010 & - & - & - & AT & $S$ & $1 \mathrm{D}$ & Terminal type \\
\hline
\end{tabular}

Identification information group

\begin{tabular}{|c|c|c|c|c|c|c|c|c|}
\hline 0008 & 0000 & 0004 & 0000 & - & $\mathrm{BD}$ & $s$ & 1 & Group length \\
\hline 0008 & 0001 & 0004 & 0000 & - & $\mathrm{BD}$ & $\mathrm{s}$ & 1 & $\begin{array}{l}\text { Message length (same as GROUP:0000, } \\
\text { ELEMENT:0001) }\end{array}$ \\
\hline 0008 & 0010 & - & - & - & AT & s & 1 & $\begin{array}{l}\text { Recognition code (used for identifying the } \\
\text { version number of this protocol) }\end{array}$ \\
\hline 0008 & 0020 & $000 \mathrm{~A}$ & 0000 & - & AT & s & 2 & $\begin{array}{l}\text { Study date (the format is yyyy.mm.dd; eg, } \\
\text { 1988.07.18) }\end{array}$ \\
\hline 0008 & 0030 & 0008 & 0000 & - & AT & s & 2 & $\begin{array}{l}\text { Study time (the format is hh:mm:ss; eg, 10: } \\
05: 03 \text { (24 hour time)) }\end{array}$ \\
\hline 0008 & 0040 & 0002 & 0000 & - & $\mathrm{BI}$ & S & 1 & $\begin{array}{l}\text { Data set type (same as GROUP:0000, } \\
\text { ELEMENT:0800) }\end{array}$ \\
\hline 0008 & 0041 & - & - & - & AT & $\mathrm{s}$ & 3 & Data set subtype \\
\hline 0008 & 0060 & 0002 & 0000 & - & AT & S & 2 & Modality (CT, NM, MR, DS, DR, US, OT) \\
\hline 0008 & 0070 & - & - & - & AT & s & 2 & Manufacturer \\
\hline 0008 & 0080 & - & - & - & AT & S & 2 & Institution ID \\
\hline 0008 & 0090 & - & - & - & AT & M & 2 & Referring physician \\
\hline 0008 & 1000 & - & - & - & AT & s & 3 & Network ID \\
\hline 0008 & 1010 & - & - & - & AT & $\mathrm{s}$ & 2 & Station ID \\
\hline
\end{tabular}


Table 1. Detailed Data Format Specification (Cont'd)

\begin{tabular}{|c|c|c|c|c|c|c|c|c|}
\hline Group & Element & & & Value & VR & VM & Type & Descripti \\
\hline \multicolumn{9}{|c|}{ Identification information group: (continued) } \\
\hline 0008 & 1030 & - & - & - & AT & $\mathbf{s}$ & 3 & Procedure description \\
\hline 0008 & 1040 & - & - & - & AT & $\mathrm{s}$ & 3 & Institutional department \\
\hline 0008 & 1050 & - & - & - & AT & M & 3 & Attending physician \\
\hline 0008 & 1060 & - & - & - & AT & $M$ & 3 & Radiologist \\
\hline 0008 & 1070 & - & - & - & AT & $M$ & 3 & Operator identification \\
\hline 0008 & 1080 & - & - & - & AT & $M$ & 3 & Admitting diagnosis \\
\hline 0008 & 1090 & - & - & - & AT & $\mathrm{s}$ & 3 & Manufacturer model \\
\hline 0008 & 4000 & - & - & - & AT & $M$ & 3 & Comments \\
\hline
\end{tabular}

General information group

$\begin{array}{lllllllll}0009 & 0000 & 0004 & 0000 & - & \text { BD } & \text { S } & 1 & \begin{array}{l}\text { Group length } \\ 0009\end{array} \\ 0000 & - & - & - & \text { AT } & \text { S } & 3 & \text { Name of file containing this message } \\ 0009 & 8010 & - & - & - & \text { AT } & \text { S } & 3 & \text { Name of file that gave rise to this file } \\ 0009 & 8020 & - & - & - & \text { AT } & \text { S } & 3 & \begin{array}{l}\text { Description of data in file } \\ \text { Scratch pad (to store any user-specified in- } \\ \text { formation, eg, measurements made on } \\ \text { the data, details of modifications made to } \\ \text { the data, etc) }\end{array}\end{array}$

Patient information group

$\begin{array}{lllllllll}0010 & 0000 & 0004 & 0000 & - & \text { BD } & \text { S } & 1 & \text { Group length } \\ 0010 & 0010 & - & - & - & \text { AT } & \text { S } & 2 & \text { Patient name } \\ 0010 & 0020 & - & - & - & \text { AT } & \text { S } & 2 & \text { Patient ID } \\ 0010 & 0030 & - & - & - & \text { AT } & \text { S } & 2 & \text { Patient birthdate } \\ 0010 & 0040 & - & - & - & \text { AT } & \text { S } & 2 & \text { Patient sex } \\ 0010 & 1000 & - & - & - & \text { AT } & \text { M } & 3 & \text { Other patient IDs } \\ 0010 & 1001 & - & - & - & \text { AT } & \text { M } & 3 & \text { Other patient name } \\ 0010 & 1005 & - & - & - & \text { AT } & \text { S } & 3 & \text { Patient's maiden name } \\ 0010 & 1010 & - & - & - & \text { AT } & \text { S } & 3 & \text { Patient age } \\ 0010 & 1020 & - & - & - & \text { AT } & \text { S } & 3 & \text { Patient size } \\ 0010 & 1030 & - & - & - & \text { AT } & \text { S } & 3 & \text { Patient weight } \\ 0010 & 1040 & - & - & - & \text { AT } & \text { S } & 3 & \text { Patient address } \\ 0010 & 1050 & - & - & - & \text { AT } & \text { M } & 3 & \text { Insurance plan ID } \\ 0010 & 1060 & - & - & - & \text { AT } & \text { S } & 3 & \text { Patient's mother's maiden name } \\ 0010 & 4000 & - & - & - & \text { AT } & \text { M } & 3 & \text { Comments }\end{array}$

Acquisition information group

$\begin{array}{lllllllll}0018 & 0000 & 0004 & 0000 & - & \text { BD } & \text { S } & 1 & \text { Group length } \\ 0018 & 0010 & - & - & - & \text { AT } & \text { M } & 2 D & \text { Contrast/bolus agent } \\ 0018 & 0020 & - & - & - & \text { AT } & \text { M } & 2 & \text { Scanning sequence } \\ 0018 & 0030 & - & - & - & \text { AT } & \text { M } & 2 & \text { Radionuclide } \\ 0018 & 0040 & - & - & - & \text { AN } & \text { S } & 2 D & \text { Cine rate } \\ 0018 & 0050 & - & - & - & \text { AN } & \text { S } & 2 & \text { Slice thickness } \\ 0018 & 0060 & - & - & - & \text { AN } & \text { M } & 2 & \text { KVP } \\ 0018 & 0070 & - & - & - & \text { AN } & \text { S } & 2 & \text { Counts accumulated } \\ 0018 & 0080 & - & - & - & \text { AN } & \text { S } & 2 & \text { Repetition time } \\ 0018 & 0081 & - & - & - & \text { AN } & \text { S } & 2 & \text { Echo time } \\ 0018 & 0082 & - & - & - & \text { AN } & \text { S } & 2 & \text { Inversion time } \\ 0018 & 0083 & - & - & - & \text { AN } & \text { S } & 2 & \text { Number of averages } \\ 0018 & 0084 & - & - & - & \text { AN } & \text { S } & 2 & \text { Imaging frequency } \\ 0018 & 0085 & - & - & - & \text { AT } & \text { S } & 2 & \text { Imaged nucleus } \\ 0018 & 1000 & - & - & - & \text { AT } & \text { S } & 3 & \text { Device serial number } \\ 0018 & 1010 & - & - & - & \text { AT } & \text { S } & 3 & \text { Film scanner ID }\end{array}$


Table 1. Detailed Data Format Specification (Cont'd)

\begin{tabular}{llllllll}
\hline Group & Element & Length & Value & VR & VM & Type & Description \\
\hline
\end{tabular}

Acquisition information group: (continued)

\begin{tabular}{|c|c|c|c|c|c|c|c|c|}
\hline 0018 & 1020 & - & - & - & AT & $\mathrm{s}$ & 3 & Software version \\
\hline 0018 & 1030 & - & - & - & AT & $\mathrm{S}$ & 3 & Protocol \\
\hline 0018 & 1040 & 一 & - & 一 & AT & $M$ & 3 & Contrast/bolus route \\
\hline 0018 & 1041 & - & - & - & AN & M & 3 & Contrast/bolus volume \\
\hline 0018 & 1042 & - & - & 一 & AT & $M$ & 3 & Contrast/bolus start time \\
\hline 0018 & 1043 & - & - & 一 & AT & $M$ & 3 & Contrast/bolus stop time \\
\hline 0018 & 1044 & - & - & - & AN & $M$ & 3 & Contrast/bolus total dose \\
\hline 0018 & 1050 & - & - & - & AN & M & 3 & Spatial resolution \\
\hline 0018 & 1060 & 一 & - & - & AN & $s$ & 3 & Trigger time \\
\hline 0018 & 1070 & - & - & - & AT & $M$ & 3 & Radionuclide route \\
\hline 0018 & 1071 & - & - & - & AN & $M$ & 3 & Radionuclide volume \\
\hline 0018 & 1072 & - & - & 一 & AT & M & 3 & Radionuclide start time \\
\hline 0018 & 1073 & - & - & - & AT & $M$ & 3 & Radionuclide stop time \\
\hline 0018 & 1074 & - & - & - & AN & $M$ & 3 & Radionuclide total dose \\
\hline 0018 & 1100 & - & - & - & AN & $\mathrm{s}$ & 3 & Reconstruction diameter \\
\hline 0018 & 1110 & - & - & 一 & AN & s & 3 & Distance source to detector \\
\hline 0018 & 1111 & - & - & - & AN & s & 3 & Distance source to patient \\
\hline 0018 & 1120 & - & - & - & AN & s & 3 & Gantry tilt \\
\hline 0018 & 1130 & - & - & - & AN & s & 3 & Table height \\
\hline 0018 & 1140 & - & - & 一 & AT & S & 3 & Rotation direction \\
\hline 0018 & 1150 & - & - & - & AN & $s$ & 3 & Exposure time \\
\hline 0018 & 1151 & - & - & 一 & AN & $s$ & 3 & Exposure rate \\
\hline 0018 & 1152 & - & - & 一 & AN & $s$ & 3 & Exposure \\
\hline 0018 & 1160 & - & - & - & AT & $M$ & 3 & Filter type \\
\hline 0018 & 1170 & - & - & - & AN & $s$ & 3 & Generator power \\
\hline 0018 & 1180 & - & - & - & AT & $M$ & 3 & Collimator/grid \\
\hline 0018 & 1190 & - & - & 一 & AN & $M$ & 3 & Focal spot \\
\hline 0018 & 1200 & - & - & - & AT & $M$ & 3 & Date of last calibration \\
\hline 0018 & 1201 & - & - & - & AT & $M$ & 3 & Time of last calibration \\
\hline 0018 & 1210 & - & - & - & AT & $M$ & 3 & Convolution kernel \\
\hline 0018 & 1240 & - & - & - & AN & $M$ & 3 & Upper/lower pixel values \\
\hline 0018 & 1242 & - & - & - & AN & $\mathrm{S}$ & 3 & Termination time \\
\hline 0018 & 1243 & - & - & - & AN & $s$ & 3 & Count rate \\
\hline 0018 & 1250 & - & - & - & AT & $s$ & 3 & Receiving coil \\
\hline 0018 & 1251 & - & - & - & AT & $s$ & 3 & Transmitting coil \\
\hline 0018 & 1260 & - & - & - & AT & s & 3 & Screen type \\
\hline 0018 & 1261 & - & - & - & AT & $\mathrm{s}$ & 3 & Phosphor type \\
\hline 0018 & 4000 & - & - & - & AT & $M$ & 3 & Comments \\
\hline
\end{tabular}

Relationship information group

$\begin{array}{lllllllll}0020 & 0000 & 0004 & 0000 & - & \text { BD } & \text { S } & 1 & \text { Group length } \\ 0020 & 0010 & - & - & - & \text { AN } & \text { S } & 2 & \text { Study (exam number) } \\ 0020 & 0011 & - & - & - & \text { AN } & \text { S } & 2 D & \text { Series } \\ 0020 & 0012 & - & - & - & \text { AN } & \text { S } & 2 D & \text { Acquisition } \\ 0020 & 0013 & - & - & - & \text { AN } & \text { S } & 2 D & \text { Image number } \\ 0020 & 0020 & - & - & - & \text { AT } & \text { M } & 2 & \text { Patient orientation } \\ 0020 & 0030 & - & - & - & \text { AN } & \text { M } & 2 & \text { Image position } \\ 0020 & 0035 & - & - & - & \text { AN } & \text { M } & 2 D & \text { Image orientation } \\ 0020 & 0050 & - & - & - & \text { AN } & \text { S } & 2 & \text { Location } \\ 0020 & 0060 & - & - & - & \text { AT } & \text { S } & 2 D & \text { Laterality } \\ 0020 & 0070 & - & - & - & \text { AT } & \text { S } & 2 D & \text { Image geometry type } \\ 0020 & 0080 & - & - & - & \text { AT } & \text { M } & 2 D & \text { Masking image } \\ 0020 & 1000 & - & - & - & \text { AN } & \text { S } & 3 & \text { No. of series in this study } \\ 0020 & 1001 & - & - & - & \text { AN } & \text { S } & 3 & \text { No. of acquisitions in this series } \\ 0020 & 1002 & - & - & - & \text { AN } & \text { S } & 3 & \text { No. of images from this acquisition }\end{array}$


Table 1. Detailed Data Format Specification (Cont'd)

\begin{tabular}{|c|c|c|c|c|c|c|c|c|}
\hline Group & Element & \multicolumn{2}{|c|}{ Length } & Value & VR & VM & Type & Description \\
\hline \multicolumn{9}{|c|}{ Relationship information group: (continued) } \\
\hline 0020 & 1020 & - & - & - & AT & M & 3 & Reference \\
\hline 0020 & 1040 & - & - & - & AT & $\mathrm{S}$ & 3 & Position reference indicator \\
\hline 0020 & 1041 & - & - & - & AN & s & 3 & Slice location \\
\hline 0020 & 1070 & - & - & - & AN & M & 3 & Other study numbers \\
\hline 0020 & $\begin{array}{c}3100 \text { to } \\
31 \mathrm{FF}\end{array}$ & - & - & - & AT & M & 2D & Source image IDs \\
\hline 0020 & 3401 & - & - & - & AT & M & 2D & Modifying device ID \\
\hline 0020 & 3402 & - & - & - & AT & $M$ & 2D & Modified image ID \\
\hline 0020 & 3403 & - & - & - & AT & $M$ & 2D & Modified image date \\
\hline 0020 & 3404 & - & - & - & AT & M & 2D & Modifying device manufacturer \\
\hline 0020 & 3405 & - & - & - & AT & M & 2D & Modified imaging time \\
\hline 0020 & 3406 & - & - & - & AT & M & 2D & Modified image description \\
\hline 0020 & 4000 & - & - & - & AT & M & 3 & Comments \\
\hline
\end{tabular}

Image presentation information group

\begin{tabular}{|c|c|c|c|c|c|c|c|c|}
\hline 0028 & 0000 & 0004 & 0000 & - & $B D$ & s & 1 & Group length \\
\hline 0028 & 0005 & 0002 & 0000 & - & $\mathrm{BI}$ & s & 1D & Image dimensions \\
\hline 0028 & 0010 & 0002 & 0000 & - & $\mathrm{BI}$ & s & 1 & No. of rows \\
\hline 0028 & 0011 & 0002 & 0000 & - & BI & $\mathrm{S}$ & 1 & No. of columns \\
\hline 0028 & 0030 & - & - & - & AN & $M$ & 2 & Pixel size \\
\hline 0028 & 0040 & - & - & - & AT & $\mathrm{s}$ & $1 \mathrm{D}$ & Image format \\
\hline 0028 & 0050 & - & - & - & AT & $M$ & 20 & Manipulated image \\
\hline 0028 & 0060 & - & - & - & AT & $\mathrm{s}$ & $1 \mathrm{D}$ & Compression code \\
\hline 0028 & 0100 & 0002 & 0000 & - & $\mathrm{Bl}$ & s & $1 \mathrm{D}$ & Bits allocated \\
\hline 0028 & 0101 & 0002 & 0000 & - & $\mathrm{Bl}$ & s & $1 \mathrm{D}$ & Bits stored \\
\hline 0028 & 0102 & 0002 & 0000 & - & $\mathrm{BI}$ & $s$ & $1 \mathrm{D}$ & High bit \\
\hline 0028 & 0104 & 0002 & 0000 & - & $\mathrm{Bl}$ & s & $2 \mathrm{D}$ & Smallest pixel value \\
\hline 0028 & 0105 & 0002 & 0000 & - & $\mathrm{BI}$ & s & $2 \mathrm{D}$ & Largest pixel value \\
\hline 0028 & 0200 & 0002 & 0000 & - & $\mathrm{Bl}$ & $\mathrm{s}$ & 10 & Image location \\
\hline 0028 & 1050 & - & - & - & AN & $M$ & 3 & Window center \\
\hline 0028 & 1051 & - & - & - & AN & $M$ & 3 & Window width \\
\hline 0028 & 1052 & - & - & - & AN & $M$ & 3 & Rescale intercept \\
\hline 0028 & 1053 & - & - & - & AN & $M$ & 3 & Rescale slope \\
\hline 0028 & 1080 & - & - & - & AT & $\mathrm{s}$ & 3 & Gray scale \\
\hline 0028 & 1100 & - & - & - & $\mathrm{BI}$ & $M$ & $2 \mathrm{D}$ & $\begin{array}{l}\text { Lookup table descriptors; gray [no. of en- } \\
\text { tries in the table (net) \starting pixel value } \\
\text { (spv) \number of bits for entries] }\end{array}$ \\
\hline 0028 & 1101 & - & - & - & $\mathrm{BI}$ & $M$ & $2 \mathrm{D}$ & Lookup table descriptors; red (as for gray) \\
\hline 0028 & 1102 & - & - & - & $\mathrm{BI}$ & M & $2 \mathrm{D}$ & Lookup table descriptors; green (as for gray) \\
\hline 0028 & 1103 & - & - & - & $\mathrm{Bl}$ & M & $2 \mathrm{D}$ & Lookup table descriptors; blue (as for gray) \\
\hline 0028 & 1200 & - & - & - & B! & M & 2D & $\begin{array}{l}\text { Lookup data; gray (mapped value } \\
1 \backslash \text { mapped value } 2 \backslash \ldots \text { mapped value } \\
\text { net; pixel values to be mapped are as- } \\
\text { sumed to be spv, spv }+1, \ldots, \text { spv }+ \text { net } \\
-1 \text {; each mapped value is stored in } 16 \\
\text { bits) }\end{array}$ \\
\hline 0028 & 1201 & - & - & - & $\mathrm{BI}$ & M & 20 & Lookup data; red (as for gray) \\
\hline 0028 & 1202 & - & - & - & Bl & M & 2D & Lookup data; green (as for gray) \\
\hline 0028 & 1203 & - & - & - & $\mathrm{Bl}$ & M & $2 \mathrm{D}$ & Lookup data; blue (as for gray) \\
\hline 0028 & 4000 & - & - & - & AT & $M$ & 3 & Comments \\
\hline
\end{tabular}

Scene-related information group*

$\begin{array}{lllllllll}0029 & 0000 & 0004 & 0000 & - & \text { BD } & \text { S } & 1 & \text { Group length } \\ 0029 & 8000 & 0002 & 0000 & - & \text { BI } & \text { S } & 1 & \text { Scene dimension (sd) }\end{array}$


Table 1. Detailed Data Format Specification (Cont'd)

\begin{tabular}{|c|c|c|c|c|c|c|c|}
\hline Group & Element & Length & Value & VR & VM & Type & Description \\
\hline
\end{tabular}

Scene-related information group: (continued)

$$
-
$$

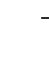

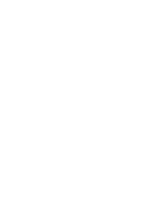

\begin{tabular}{|c|c|c|c|c|c|c|}
\hline 0029 & 8015 & - & - & - & AT & $M$ \\
\hline 0029 & 8020 & - & - & - & $\mathrm{Bl}$ & $M$ \\
\hline 0029 & 8030 & 0002 & 0000 & - & $\mathrm{BI}$ & $\mathrm{s}$ \\
\hline 0029 & 8040 & - & - & - & AN & $M$ \\
\hline 0029 & 8050 & - & - & - & AN & $M$ \\
\hline 0029 & 8060 & 0002 & 0000 & - & $\mathrm{BI}$ & $S$ \\
\hline 0029 & 8070 & - & - & - & $\mathrm{BI}$ & $M$ \\
\hline 0029 & 8080 & 0002 & 0000 & - & $\mathrm{BI}$ & $S$ \\
\hline 0029 & 8090 & - & - & - & $\mathrm{BI}$ & $M$ \\
\hline
\end{tabular}

AN $\quad M \quad 1 D$

Location and orientation of the scene domain with respect to a fixed imaging device coordinate system: specified by $(s d+1)$ vectors $X_{0}, \ldots, X_{s d} . X_{0}$ : coordinates of the origin of the scene coordinate system. $X_{1}, \ldots, X_{s d}$ : unit vectors along each of its sd principal directions

Labels associated with the axes

Unit of measurement in the sd directions: 0 . $\mathrm{km} ; 1, \mathrm{~m} ; 2, \mathrm{~cm} ; 3, \mathrm{~mm} ; 4, \mu \mathrm{m} ; 5, \mathrm{~s} ; 6$, $\mathrm{ms} ; 7, \mu \mathrm{s}$

No. of density values associated with each cell (nd)

Smallest density value (when density is vector valued, the smallest of each component in the scene)

Largest density value (when density is vector valued, the largest of each component in the scene)

Density storage scheme: number of integers in the density vector (ni). (The rest nd-ni are assumed to be floating-point numbers)

Density storage scheme: whether each integer component is signed or unsigned: sous $\backslash \backslash$. . \sousni $\backslash$, where each entry indicates whether each integer component of the density vector is signed (1) or unsigned (0)

Density storage scheme: total bits required for the density vector (nbi)

Density storage scheme:

lbit $1 \backslash$ rbit $1 \backslash$. . \lbitnd \rbitnd $\backslash$. Within the bit field of nbi bits for the density vector, the bits are numbered $0,1, \ldots$, nbi- 1 from left to right (most significant to least significant) and the bit field for each component is specified by a left bit (lbit) and a right bit (rbit). Thus, for every component lbit $\leq$ rbit. The lbit, rbit pair for a floating point number indicates whether the number is of single or double precision (see the definition of value representation GN in Section 2). The density vector itself is stored as integer $1 \backslash$ integer $2 \backslash$

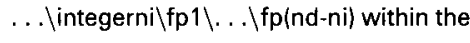
$\mathrm{nbi}$ bits bit field and the order of the bit fields is preserved in the file storing the message.

Size of each slice: null (ie, LENGTH $=0$ ) for IMAGE 1 type; for IMAGE0 type this element stores no. of columns $\backslash$ no. of rows $\backslash$ in the slice

Sampling scheme (no. of n-scenes): null (ie, LENGTH $=0$ ) for IMAGE1 type. For IMAGE0 type, this element stores, for each $n, 2<n \leq s d$, the number of (n - 1)-scenes for each n-scene. These numbers are stored in the order $\mathrm{ns} 0 \backslash \mathrm{ns} 00 \backslash \mathrm{ns} 01 \backslash \mathrm{ns} 02 \backslash \ldots$ \ns000 \ns001\. . . (See IMAGE0) 
Table 1. Detailed Data Format Specification (Cont'd)

\begin{tabular}{llllllll}
\hline Group & Element & Length & Value & VR & VM & Type & \\
\hline
\end{tabular}

Scene-related information group: (continued)

\begin{tabular}{|c|c|c|c|c|c|c|c|c|}
\hline 0029 & $80 A 5$ & - & - & - & AN & $\mathrm{M}$ & 1 & $\begin{array}{l}\text { Pixel size in the } x_{1} \text { and } x_{2} \text { directions: } \\
\text { psizex } 1 \backslash p \text { sizex } 2\end{array}$ \\
\hline 0029 & $80 \mathrm{BO}$ & - & - & - & AN & $M$ & 1 & 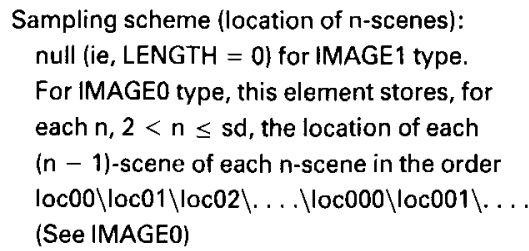 \\
\hline 0029 & $80 \mathrm{CO}$ & - & - & - & $A T$ & $M$ & 3 & $\begin{array}{l}\text { Description of processing operations on this } \\
\text { scene and their parameters: type of pro- } \\
\text { cessing (filtering, segmentation, interpola- } \\
\text { tion, classification, masking, reslicing, his- } \\
\text { togram transforms, ROI), methods, } \\
\text { parameters of the method }\end{array}$ \\
\hline
\end{tabular}

Structure-related information group $\dagger$

\begin{tabular}{|c|c|c|c|c|c|c|c|c|}
\hline $002 B$ & 0000 & 0004 & 0000 & - & $\mathrm{BD}$ & $\mathbf{S}$ & 1 & Group length \\
\hline DO2B & 8000 & 0002 & 0000 & - & B! & $\mathrm{S}$ & 1 & Structure dimension (std) \\
\hline $002 B$ & 8010 & - & - & - & AN & M & $1 \mathrm{D}$ & $\begin{array}{l}\text { Location and orientation of the structure co- } \\
\text { ordinate system with respect to the imag- } \\
\text { ing device coordinate system: specified } \\
\text { by std }+1 \text { vectors } Y_{0,}, Y_{1}, \ldots, Y_{s t}, Y_{0} ; \text { coor- } \\
\text { dinates of the origin of the structure coor- } \\
\text { dinate system. } Y_{1} \ldots, Y_{\text {std }} \text { : unit vectors } \\
\text { along each of its std principal directions }\end{array}$ \\
\hline 0028 & 8015 & - & - & - & AT & M & 2D & Labels associated with the axes \\
\hline $002 B$ & 8020 & - & - & 一 & BI & $M$ & $1 \mathrm{D}$ & $\begin{array}{l}\text { Unit of measurement in the std directions: } 0 \text {, } \\
\mathrm{km} ; 1, \mathrm{~m} ; 2, \mathrm{~cm} ; 3, \mathrm{~mm} ; 4, \mu \mathrm{m} ; 5, \mathrm{~s} ; 6 \text {, } \\
\mathrm{ms} ; 7, \mu \mathrm{s}\end{array}$ \\
\hline $002 B$ & 8060 & 0002 & 0000 & - & $\mathrm{BI}$ & $S$ & 1 & $\begin{array}{l}\text { No. of components in the vector NTSE as- } \\
\text { signed to each nonterminal structure ele- } \\
\text { ment (ncnt) }\end{array}$ \\
\hline $002 B$ & 8070 & - & - & - & AN & $M$ & $1 \mathrm{D}$ & $\begin{array}{l}\text { Smallest value of each component of TSE in } \\
\text { the structure }\end{array}$ \\
\hline 002B & 8080 & - & - & - & AN & $M$ & $1 \mathrm{D}$ & $\begin{array}{l}\text { Largest value of each component of TSE in } \\
\text { the structure }\end{array}$ \\
\hline $002 B$ & 8090 & 0002 & 0000 & - & $\mathrm{BI}$ & $S$ & 1 & $\begin{array}{l}\text { Storage for terminal structure elements: no. } \\
\text { of integers in TSE (nit). (The rest of the } \\
\text { nct-nit components are floating point } \\
\text { numbers) }\end{array}$ \\
\hline 0028 & 8080 & 0002 & 0000 & - & BI & $S$ & 1 & $\begin{array}{l}\text { Storage scheme for terminal structure ele- } \\
\text { ments: total bits required for TSE (nbit) }\end{array}$ \\
\hline
\end{tabular}


Table 1. Detailed Data Format Specification (Cont'd)

\begin{tabular}{|c|c|c|c|c|c|c|c|c|}
\hline Group & Element & \multicolumn{2}{|c|}{ Length } & Value & VR & VM & Type & Description \\
\hline \multicolumn{9}{|c|}{ Structure-related information group: (continued) } \\
\hline 0028 & $80 \mathrm{CO}$ & - & - & - & 81 & M & 1 & $\begin{array}{l}\text { Storage scheme for TSE: } \\
\text { Ibit } 1 \backslash \text { rbit } 1 \backslash \ldots \text {. . Ibitnct } \backslash \text { rbitnct } \backslash \text {. Descrip- } \\
\text { tion is similar to that for GROUP:0029, } \\
\text { ELEMENT:8090. If Ibit > rbit, it means that } \\
\text { the component of TSE associated with } \\
\text { this field is not stored }\end{array}$ \\
\hline $002 B$ & 8000 & 0002 & 0000 & - & $\mathrm{BI}$ & $\mathrm{s}$ & 1 & $\begin{array}{l}\text { Storage scheme for nonterminal structure } \\
\text { elements: no. of integers in NTSE (nint). } \\
\text { (The rest of the ncnt-nint components are } \\
\text { floating point numbers) }\end{array}$ \\
\hline $002 B$ & $80 \mathrm{E} 0$ & - & 一 & - & $\mathrm{BI}$ & M & $1 \mathrm{D}$ & $\begin{array}{l}\text { Whether each integer component of NTSE is } \\
\text { signed or unsigned: } \\
\text { sous } 1 \backslash \text { sous } 2 \backslash . . \text { ।sousnint } \backslash \text {, where each } \\
\text { entry indicates whether each integer com- } \\
\text { ponent of NTSE is signed (1) or unsigned } \\
\text { (0) }\end{array}$ \\
\hline 0028 & $80 F 0$ & 0002 & 0000 & - & $\mathrm{Bl}$ & s & 1 & $\begin{array}{l}\text { Storage scheme for nonterminal structure } \\
\text { elements: no. of bits required for NTSE } \\
\text { (nbint) }\end{array}$ \\
\hline 0028 & 8100 & - & - & - & BI & M & 1 & 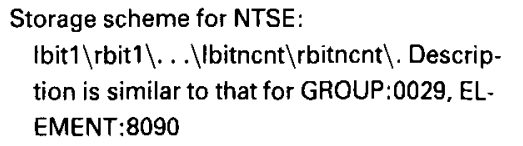 \\
\hline 002B & 8110 & - & - & - & $\mathrm{Bl}$ & $M$ & 1 & $\begin{array}{l}\text { Sampling scheme for the structure: this ele- } \\
\text { ment stores, for each } \mathrm{n}, 2<\mathrm{n} \leq \mathrm{std} \text {, the } \\
\text { number of samples in each dimension. } \\
\text { These numbers are stored in the order } \\
\mathrm{ns} 0 \backslash \mathrm{ns} 00 \backslash \mathrm{ns} 01 \backslash \mathrm{ns} 02 \backslash \ldots \text { ns } 000 \backslash \mathrm{ns} 001 \backslash \ldots \\
\text { (see previous section) }\end{array}$ \\
\hline D02B & 8120 & - & - & - & AN & $M$ & 1 & $\begin{array}{l}\text { Sample size in the } y_{1} \text { and } y_{2} \text { directions: } \\
\text { ssizey } 1 \backslash \text { ssizey2 }\end{array}$ \\
\hline 002B & 8130 & - & - & - & AN & $M$ & 1 & $\begin{array}{l}\text { Sampling scheme for the structure: This ele- } \\
\text { ment stores, for each } n, 2<n \leq \text { std, the } \\
\text { sample location in the order } \\
\text { loc } 00 \backslash \text { loc } 01 \text { loc } 02 \backslash \ldots \text { । loc } 000 \backslash \text { loc } 001 \backslash \ldots \\
\text { (see previous section) }\end{array}$ \\
\hline 002B & 8140 & - & - & - & AN & $M$ & 2 & 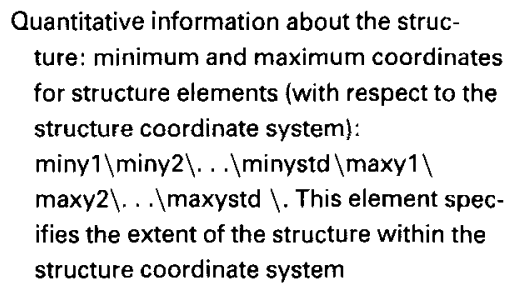 \\
\hline $002 B$ & 8150 & - & - & - & AN & $S$ & 3 & $\begin{array}{l}\text { Quantitative information about the struc- } \\
\text { ture: volume }\end{array}$ \\
\hline D02B & 8160 & - & - & - & AN & $S$ & 3 & $\begin{array}{l}\text { Quantitative information about the struc- } \\
\text { ture: surface area }\end{array}$ \\
\hline O02B & 8170 & - & - & - & AN & $S$ & 3 & $\begin{array}{l}\text { Quantitative information about the struc- } \\
\text { ture: rate of change of volume }\end{array}$ \\
\hline $002 B$ & 8180 & - & - & - & AT & $M$ & 3 & $\begin{array}{l}\text { Description of structure extraction method } \\
\text { and its parameters }\end{array}$ \\
\hline
\end{tabular}


Table 1. Detailed Data Format Specification (Cont'd)

\begin{tabular}{|c|c|c|c|c|c|c|c|c|}
\hline Group & Element & \multicolumn{2}{|c|}{ Length } & Value & VR & VM & Type & Description \\
\hline \multicolumn{9}{|c|}{ Display-related information group $\ddagger$} \\
\hline $002 D$ & 0000 & 0004 & 0000 & - & $B D$ & $\mathrm{~s}$ & 1 & Group length \\
\hline 002D & 8000 & 0002 & 0000 & - & $\mathrm{Bl}$ & S & 1 & Dimensionality of the display data (ddd) \\
\hline 002D & 8010 & 0004 & 0000 & - & $\mathrm{Bl}$ & M & 1D & $\begin{array}{l}\text { Unit of measurement for the pixel size: } 0 \\
\mathrm{~km} ; 1, \mathrm{~m} ; 2, \mathrm{~cm} ; 3, \mathrm{~mm} ; 4, \mu \mathrm{m} ; 5, \mathrm{~s} ; 6 \\
\mathrm{~ms} ; 7, \mu \mathrm{s}\end{array}$ \\
\hline $002 \mathrm{D}$ & 8020 & 0002 & 0000 & - & $\mathrm{Bl}$ & S & 1 & $\begin{array}{l}\text { Intensity storage scheme: no. of elements in } \\
\text { the intensity vector associated with the } \\
\text { pixels (neiv) }\end{array}$ \\
\hline 002D & 8030 & - & - & - & AN & M & 1D & $\begin{array}{l}\text { Smallest value of each component of the } \\
\text { intensity vector in the display }\end{array}$ \\
\hline $002 D$ & 8040 & - & - & - & AN & M & $1 \mathrm{D}$ & $\begin{array}{l}\text { Largest value of each component of the in- } \\
\text { tensity vector in the display }\end{array}$ \\
\hline $002 \mathrm{D}$ & 8050 & 0002 & 0000 & - & $\mathrm{BI}$ & S & 1 & $\begin{array}{l}\text { Intensity storage scheme: no. of integer- } \\
\text { valued elements in the intensity vector } \\
\text { (niiv). (The rest of the neiv-niiv elements } \\
\text { are assumed to be floating point numbers) }\end{array}$ \\
\hline 002D & 8060 & - & - & - & $\mathrm{BI}$ & M & 10 & $\begin{array}{l}\text { Whether each of the integer-valued ele- } \\
\text { ments in the intensity vector is signed or } \\
\text { unsigned: sous } 1 \backslash . . \text { Isousniiv } \backslash \text {, where } \\
\text { each entry indicates whether each integer } \\
\text { component of the intensity vector is } \\
\text { signed (1) or unsigned (0) }\end{array}$ \\
\hline 002D & 8070 & 0002 & 0000 & - & BI & s & 1 & $\begin{array}{l}\text { Intensity storage scheme: total bits required } \\
\text { for the intensity vector (nbiiv) }\end{array}$ \\
\hline $002 D$ & 8080 & - & - & - & $\mathrm{Bl}$ & M & 1 & $\begin{array}{l}\text { Intensity storage scheme: } \\
\text { Ibit1 } 1 \text { rbit } 1 \backslash . \text {. Ibitneiv } \mathbf{r b i t n e i v} \text {. This ele- } \\
\text { ment specifies the bit field for each ele- } \\
\text { ment of the intensity vector via a left-bit, } \\
\text { right-bit pair of numbers; see GROUP: } \\
0029 \text {, ELEMENT:8090 }\end{array}$ \\
\hline 002D & 8090 & 0002 & 0000 & - & $\mathrm{Bl}$ & S & 1 & Total images in the display (tni) \\
\hline 002D & 80A0 & 0004 & 0000 & - & $\mathrm{BI}$ & M & 1 & $\begin{array}{l}\text { Size of the image: no. of columns } \backslash \text { no. of } \\
\text { rows } \backslash\end{array}$ \\
\hline $002 D$ & $80 \mathrm{BO}$ & - & - & - & AN & M & 10 & Pixel size in the $z_{1}$ and $z_{2}$ directions \\
\hline O02D & $80 \mathrm{CO}$ & - & - & - & AT & $M$ & 2 & $\begin{array}{l}\text { Specification of the meaning of each com- } \\
\text { ponent of PV: } \\
\text { meaning1 } \backslash \text { meaning2 } \backslash . . \text { (meaning(ddd-2) }\end{array}$ \\
\hline O02D & $80 D 0$ & - & - & - & BI & $M$ & 2 & $\begin{array}{l}\text { Characteristics of the images: This element } \\
\text { stores the parameter vectors that describe } \\
\text { the characteristics of the images in the } \\
\text { display: } P V 1 \backslash P V 2 \backslash \ldots \backslash P V \text { tni } \backslash\end{array}$ \\
\hline $002 \mathrm{D}$ & $80 E 0$ & - & - & - & AT & $M$ & 3 & $\begin{array}{l}\text { Description of visualization method and its } \\
\text { parameters for each object [eg, volume } \\
\text { rendering or surface rendering; ray-cast- } \\
\text { ing or projection; shading method (scene- } \\
\text { space, view space, object space); shading } \\
\text { parameters (diffuse parameters, specular } \\
\text { parameters, ambient); antialiasing } \\
\text { method; color of the object; etc] }\end{array}$ \\
\hline
\end{tabular}

Scene data group

$\begin{array}{llllllll}\text { 7FEO } & 0000 & 0004 & 0000 & - & \text { BD } & S & 1\end{array}$

Group length: A value FFFF FFFF signals that the number of bytes required for the cell density values $\geq$ FFFF FFFF and that data might be stored in subsequent oddnumbered groups $7 F E 1,7 F E 3, \ldots$, following cell density values in this group 
Table 1. Detailed Data Format Specification (Cont'd)

\begin{tabular}{|c|c|c|c|c|c|c|c|c|}
\hline Group & Element & \multicolumn{2}{|c|}{ Length } & Value & VR & VM & Type & Description \\
\hline \multicolumn{9}{|c|}{ IMAGE0 type } \\
\hline 7FE0 & 0010 & - & - & - & GN & M & 1 & $\begin{array}{l}\text { Cell densities: stored as per density storage } \\
\text { scheme, as a sequence of } n d \text {-tuples } \\
\text { CD } 1 \backslash C D 2 \backslash \ldots, \text { where CDi contains the } \\
\text { densities associated with the cell num- } \\
\text { bered } i \text {. The cells are numbered consecu- } \\
\text { tively changing the coordinates in the fol- } \\
\text { lowing order: } x_{1}, x_{2}, \ldots, x_{\text {sd }} \text { (ie, in the row- } \\
\text { by-row, slice-by-slice, etc order) }\end{array}$ \\
\hline 7FE0 & 0010 & - & - & - & GN & M & 1 & $\begin{array}{l}\text { Cell specification (locations and densities): } \\
\text { stored as per density storage scheme, as } \\
\text { a sequence of pairs LOC } 1 \backslash C D 1 \backslash L O C 2 \backslash \\
\text { CD2 } \backslash . . . \text { where LOC } i \text { is an sd-tuple indi- } \\
\text { cating the location of cell } i \text { and CD } i \text { is an } \\
\text { nd-tuple storing the densities of cell } i\end{array}$ \\
\hline \multicolumn{9}{|c|}{ Structure data group } \\
\hline 8001 & 0000 & 0004 & 0000 & - & $\mathrm{BD}$ & $\mathbf{S}$ & 1 & $\begin{array}{l}\text { Group length: A value of FFFF FFFF signals } \\
\text { that the no. of bytes required for structure } \\
\text { data is } \geq \text { FFFF FFFF and that data might } \\
\text { be stored in subsequent odd-numbered } \\
\text { groups } 8003,8005, \ldots \text {, following data in } \\
\text { this group }\end{array}$ \\
\hline 8001 & 8000 & - & - & - & GN & $M$ & 1 & $\begin{array}{l}\text { Structure data: The vectors (NTSE or TSE) } \\
\text { assigned to the nodes of the tree repre- } \\
\text { senting the structure are stored in the } \\
\text { breadth-first order of the nodes (see Sec- } \\
\text { tion 3) vector for node } 0 \backslash \text { vector for node } \\
00 \backslash \text { vector for node } 01 \backslash \ldots \text { Ivector for node } \\
000 \backslash \ldots \text { Note that, because of the nature } \\
\text { of the tree, for structures of type CURVE0, } \\
\text { SURFACE0, and SHELL, the vectors NTSE } \\
\text { come first in this element, which are then } \\
\text { followed by the vectors TSE. For other } \\
\text { general tree structures this order may not } \\
\text { occur }\end{array}$ \\
\hline \multicolumn{9}{|c|}{ Display data group } \\
\hline 8021 & 0000 & 0004 & 0000 & - & $B D$ & $\mathbf{S}$ & 1 & $\begin{array}{l}\text { Group length: A value of FFFF FFFF signals } \\
\text { that the no. of bytes required for display } \\
\text { data is } \geq \text { FFFF FFFF and that data might } \\
\text { be stored in subsequent odd-numbered } \\
\text { groups } 8023,8025, \ldots \text {, following data in } \\
\text { this group }\end{array}$ \\
\hline 8021 & 8000 & - & - & - & GN & M & 1 & $\begin{array}{l}\text { Display data: The intensity vectors (IV) are } \\
\text { stored as per intensity storage scheme as } \\
\text { a sequence of neiv tuples } \\
\text { IV } 1 \backslash I V 2 \backslash \ldots \text { where IV } i \text { contains the intensi- } \\
\text { ties associated with the pixel numbered } i \text {. } \\
\text { The pixels are numbered in the row-by- } \\
\text { row order }\end{array}$ \\
\hline
\end{tabular}

*See Scene Data for definition of terms.

tSee Structure Data.

¥See Display Data. 
GROUP:8001; display = groups up to GROUP: 0028 and GROUP:002D, GROUP:8021.

\section{LIMITATIONS AND EFFICIENCY}

The existing ACR-NEMA standards specification ${ }^{15}$ has two important limitations. First, the byte ordering specified by the standards for value representation methods denoted $\mathrm{BD}, \mathrm{AN}$, and AT is not natural for machines that consider the bytes to be in a monotonically decreasing order of significance from left to right. This calls for rearrangement of bytes whenever a standard message is to be interpreted by such machines or when they must create the message in the standard form from an internal representation. Multidimensional imaging systems can handle this conflict by gathering machinespecific byte-order information (at the time of installation of the system) and by automatically reordering bytes when needed. The loss of efficiency incurred in this process is not significant because types BD, AN, and AT occur only in the descriptive part (header) of messages.

The second limitation comes from the requirement that all floating point numbers be stored in ASCII numeric form. Although in many situations floating point numbers can be replaced by integers without much loss of information, fulfillment of this condition may not be guaranteed in general. The new value representation method called GN suggested in this protocol is an effective solution to this limitation.

Because we wanted this specification to be compatible with the existing ACR-NEMA standards, some of the groups we have created have information of somewhat similar nature to that occurring in standard groups. The specification can be made more efficient by merging relevant groups or elements from such groups. For example, image presentation and scene-related
Table 2. Storage Requirement for Some Typical Data Sets

\begin{tabular}{lrr}
\hline & Header & \multicolumn{1}{c}{ Data } \\
\hline IMAGEO & $1 \mathrm{~KB}$ & $9 \mathrm{MB}$ \\
CURVE0 & $2 \mathrm{~KB}$ & $0.3 \mathrm{MB}$ \\
SURFACE0 & $0.6 \mathrm{~KB}$ & $2.7 \mathrm{MB}$ \\
SHELL & $2.5 \mathrm{~KB}$ & $1.2 \mathrm{MB}$ \\
\hline
\end{tabular}

information groups can be merged into a single group to eliminate some of the repetitions.

Since descriptive information (headers) pertaining to scene and structure data is stored on a need basis, the additional storage required owing to the generality of the specification can be kept to a minimum. We emphasize again that for a given data type only those message items that are relevant to the data type are stored. The storage requirement figures determined for a typical data set (Table 2) give some idea of the cost of storage for the header part of the data (groups up to 002D) and for the data part (groups beyond 002D).

\section{CONCLUSIONS}

As multidimensional image processing, visualization, and analysis become more common, standard means of representing and storing image and nonimage data will be needed for convenient exchange of information, and for development of software environments that are application, data, machine, and approach independent. In this article, we have described an extension to and a generalization of the 2-D ACR-NEMA standards to meet these requirements. The generalized specification is actively being used in a software environment being developed by us, called 3DVIEWNIX, for multidimensional biomedical image display, processing, and analysis.

\section{ACKNOWLEDGMENT}

We thank Mary A. Blue for typing the manuscript.

\section{REFERENCES}

1. Stark DD, Bradley WG: Magnetic Resonance Imaging. St. Louis, MO, Mosby, 1988, pp 172-174

2. Robb RA: High-speed three-dimensional x-ray computed tomography. Proc IEEE 71:308-319, 1983

3. Gadian DG: NMR and Its Application to Living Systems. Oxford, England, Oxford University Press, 1982

4. Principe JC, Yu FS, Reid SA: Display of EEG chaotic dynamics, in Proceedings, First International Conference on Visualization in Biomedical Computing. Atlanta, GA,
1990. Los Alamitos, CA, IEEE Computer Society, 1990, pp $346-351$

5. Udupa JK, Herman GT (eds): 3D Imaging in Medicine. Boca Raton, FL, CRC 1991

6. Axel L, Dougherty L: MR imaging of motion with spatial modulation of magnetization. Radiology 171:841845,1989

7. Axel L, Goncalves R, Bloomgarden D: Two-dimensional analysis and functional imaging of regional heart wall 
motion with magnetic resonance imaging. Radiology (in press)

8. Tag Image File Format-Rev.5.0, An Aldus Corporation (Seattle, WA)/Microsoft Corporation (Redmond, WA) Technical Memorandum, 1987

9. Thomas SG: Design of the Utah RLE format. Technical Report 86-15, Alpha-1 Project, Computer Science Department, University of Utah, Salt Lake City, November 1986

10. Macintosh technical notes No. 21 (quickdraw's internal picture definition), No. 41 (drawing to an offscreen bitmap), No. 120 (drawing to an offscreen pixmap), and No. 154 (displaying large pict files), Apple Computer, Inc, Cupertino, CA

11. Treinish LA: SIGGRAPH ' 90 workshop report: Data structures and access software for scientific visualization. Computer Graphics, 25:104-118, 1991

12. Hung HM, Udupa JK, Goncalves R, et al: The 3DVIEWNIX software system-data-, graphics-, and process-interface functions-version 1.0. Technical Report MIPG178, Medical Image Processing Group, Department of Radiology, University of Pennsylvania, Philadelphia, PA, January 1991

13. Thompson KL, Ritchie DM: The UNIX time-sharing system. Communications ACM 17:365-375, 1974

14. Scheifler RW, Gettys J, Newman R: X Window System C Library and Protocol Reference. Bradford, MA, Digital, 1988

15. ACR-NEMA: Digital imaging and communications. ACR-NEMA Standards Publication No. 300-1985, Washington, DC, National Electrical Manufacturer's Association, 1985

16. Palmer JF, Morse SP: The 8087 Primer. New York, NY, Wiley 1984, pp 12-28
17. Udupa JK: A unified theory of objects and their boundaries in multidimensional digital images, in Jaffee CC, Lemke HU, Rhodes ML, et al (eds): Proceedings of Computer Assisted Radiology, CAR'87. Berlin, Germany, July 1-4, 1987, pp 779-784

18. Udupa JK, Hung HM, Chuang KS: Surface and volume rendering in 3D imaging: A comparison. J Digital Imaging 4:159-168, 1991

19. Udupa JK, Ajjanagadde VG: Boundary and object labelling in three-dimensional images. Comput Vision Graphics Image Processing 51:355-369, 1990

20. Cline HE, Lorenson WE, Ludke $S$, et al: Two algorithms for the three-dimensional reconstruction of tomograms. Med Physics 15:320-327, 1987

21. Fuchs H, Kedem ZM, Uselton SP: Optimal surface reconstruction for planar contours. Communications ACM 20:693-702, 1977

22. Boissonnat JD: Shape reconstruction from planar cross sections. Comput Vision Graphics Image Processing 44:1-29, 1988

23. Udupa JK, Odhner D: Interactive surgical planning: high-speed object rendition and manipulation without specialized hardware, in: Proceedings of the First International Conference on Visualization in Biomedical Computing. Atlanta, GA, 1990. Los Alamitos, CA, IEEE Computer Society, 1990, pp 330-336

24. Levoy M: Display of surfaces from volume data. IEEE Comput Graphics Applications 8:29-37, 1988

25. Drebin RA, Carpenter L, Hanrahan P: Volume rendering. Comput Graphics 22:65-74, 1988

26. Kaufman A: A Tutorial on Volume Visualization. Los Alamitos, CA, IEEE Computer Society Press, 1990

27. Hoehne KH, Bomans M, Pommert A, et al: 3Dvisualization of tomographic volume data using the generalized voxel model. Visual Comput 6:28-37, 1990 